\title{
Simulación de problemas probabilísticos
}

\author{
Greivin Ramírez Arce \\ gramirez@itcr.ac.cr \\ Instituto Tecnológico de \\ Costa Rica - Costa Rica
}

Recibido: Octubre 24, $2014 \quad$ Aceptado: Febrero 6, 2014

\begin{abstract}
Resumen. La simulación computacional de problemas de probabilidad permite obtener sus soluciones a través de la frecuencia relativa del número de éxitos obtenidos en los $n$ experimentos realizados. La ley de los grandes números respalda una buena aproximación de la probabilidad teórica de un evento a través de la repetición sucesiva de experimentos. A continuación se presentan una serie de problemas probabilísticos con una posible simulación realizada en los paquetes Fathom y Excel. La solución teórica de estos problemas requiere conocimientos básicos de probabilidad, por lo que las simulaciones realizadas buscan dar una propuesta de solución a estos problemas sin tener que acudir al formalismo matemático.
\end{abstract}

Palabras clave: Probabilidades, resolución de problemas, Simulación, Excel, Fathom

\begin{abstract}
Computer simulation of probability problems can get their solutions through the relative frequency of the number of successes in $n$ experiments. The law of large numbers supports a good approximation of the theoretical probability of an event through the successive repetition of experiments. Below is a series of probability problems with a possible simulation performed in Fathom or Excel packages. The theoretical solution of these problems requires a basic knowledge of probability, so the simulations seek to a proposed solution to these problems without resorting to mathematical formalism.
\end{abstract}

KeyWords: Probability, problem solving, simulation, Excell, Fathom 


\subsection{Introducción}

La simulación computacional es sugerida como estrategia metodológica que permirá la solución frecuencial de problemas de probabildad [1], [2],[3],[5] y [6].

El proceso previo de simulación de eventos puede contribuir a:

- Determinar que tan probables son individuos o las clases de individuos en los modelos de optimización para medir su inercia.

- Calcular la probabilidad de que una enfermedad se propague, así como determinar qué tan posible es que después de un tratamiento, el individuo sea susceptible a volverla a contraer.

- Conocer contextos donde se tienen pocos datos o se carece de ellos.

- Determinar la probabilidad de eventos que varían en series de tiempo.

Para el siguiente cúmulo de problemas se propone una posible simulación a través de conocimientos básicos de programación en Excel y Fathom. Además, se muestra una solución teórica con el fin de comparar lo alcanzado en la simulación con el proceso formal matemático.

Se seleccionaron los paquetes Excel y Fathom por su accesibilidad del primero y por su facilidad de muestreo del segundo.

\subsection{Problemas}

\subsubsection{Parada de autobuses}

Imagine que está esperando un autobús en una terminal que está muy saturada. Uno de cada cuatro autobuses llegan en forma aleatoria que le pueden llevar a su destino.

- ¿Cuántos autobuses crees que pueden arribar antes de que llegue el primero que se dirige a su destino?

- ¿Cuál crees que será el máximo número de autobuses que llegarán antes de que arribe el autobús que se dirige a su destino?

- ¿Cuál es la probabilidad que entre los primeros cinco autobuses que arriban se encuentre al menos uno que te lleve a tu destino?

\section{Simulación en computadora}

1. De la barra de herramientas de Fathom arrastre una colección y asígnele el nombre de Autobuses.

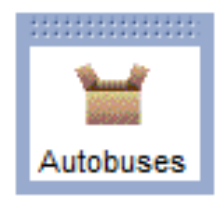

Simulación de problemas probabilísticos. Greivin Ramírez

Derechos Reservados (C) 2015 Revista digital Matemática, Educación e Internet (http://tecdigital.tec.ac.cr/revistamatematica/) 
2. Arrastre la tabla correspondiente y asígnele el nombre Autobuses al atributo y escriba directamente tres veces 0 y una vez 1 .

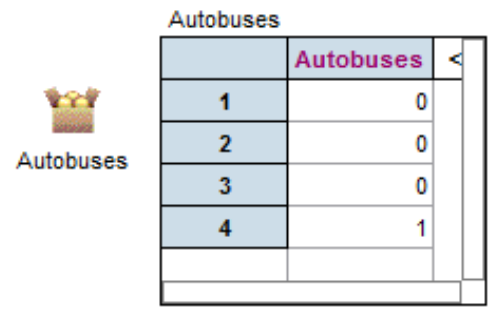

3. Seleccione la caja Autobuses haciendo clic sobre ella y del menú Colección seleccione Muestrear casos. Automáticamente aparece otra caja con la etiqueta Muestra de Autobuses.

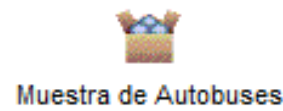

4. Active el inspector haciendo doble clic en la caja de Muestra de Autobuses y modifíquelo como se muestra a continuación. Por omisión el programa toma una muestra de tamaño 10, pero se puede cambiar, ya sea modificando el número de casos o seleccionando la condicional has ta la condición, como lo hacemos a continuación:

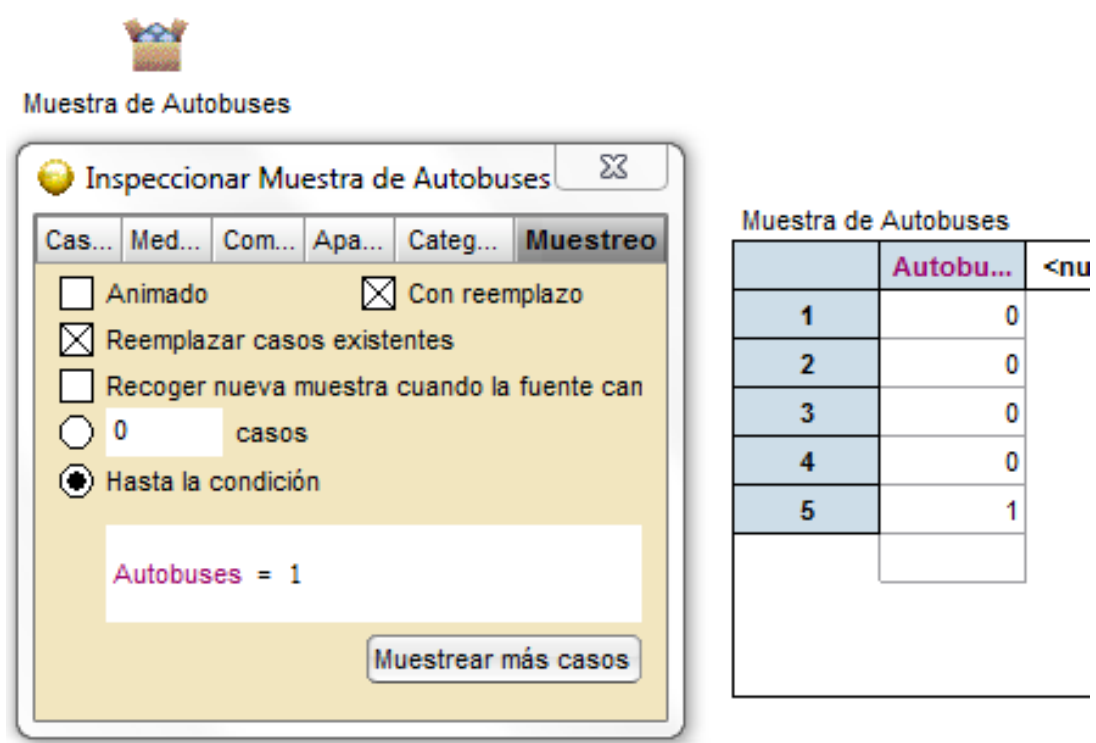

5. Entre a la pestaña Medidas y defina la medida No_Autobuses y con doble clic en la casilla de fórmula activa al editor y escribe la fórmula count ( ) -1, como se muestra enseguida: 


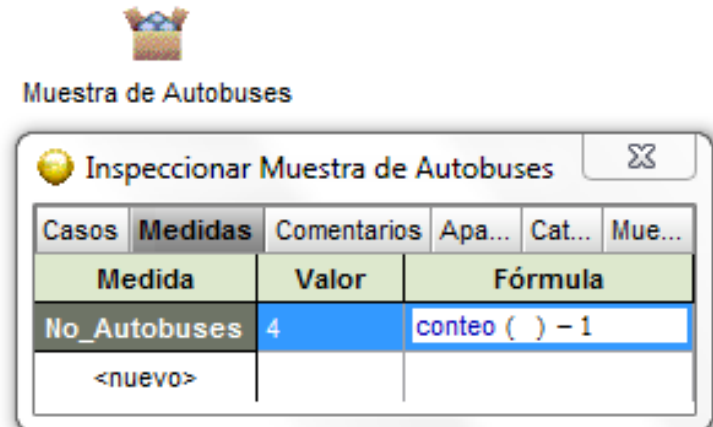

Muestra de Autobuses
\begin{tabular}{|c|r|}
\hline & Autobu... \\
\hline 1 & 0 \\
\hline 2 & 0 \\
\hline 3 & 0 \\
\hline 4 & 0 \\
\hline 5 & 1 \\
\hline
\end{tabular}

Esto permitirá contar el número de autobuses incorrectos que llegan antes del autobús correcto por eso restamos 1 .

6. Activar el icono Muestra de Autobuses y del menú Colección elige Recoger medidas. Aparecerá entonces una nueva caja denominada Medidas de Muestra de Autobuses.

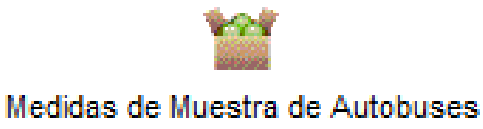

7. Arrastre y despliegue la tabla correspondiente. Modifique el inspector haciendo doble clic sobre la caja Medidas de Muestra de Autobuses y escriba 1000 casos en lugar de 5, desactive la animación y presione Collect More Measures.

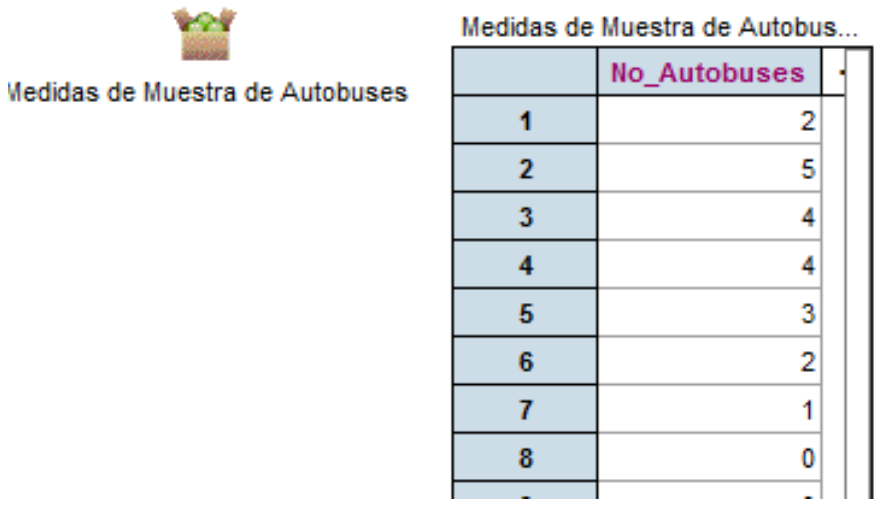

8. Para construir un diagrama de barras con los datos anteriores, active el ícono de gráfica hacia la hoja de trabajo y arrastre No_ Autobuses hacia el eje horizontal de la gráfica dejando presionada la tecla shift. En seguida aparecerá una gráfica similar a la que se muestra enseguida. 


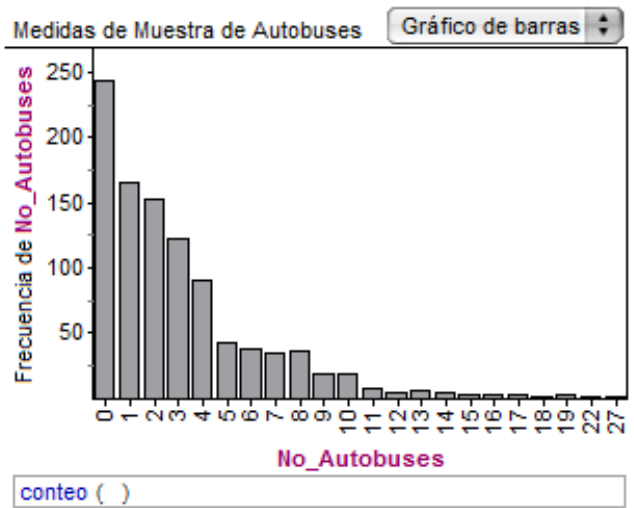

9. Arrastre el ícono de la tabla resumen hacia la hoja de trabajo. Luego coloque hacia ella el atributo No_Autobuses dejando presionado la tecla shift a la vez. La tabla muestra el total de autobuses que llegaron antes de que llegara el autobús correcto.

Medidas de Muestra de Autobuses

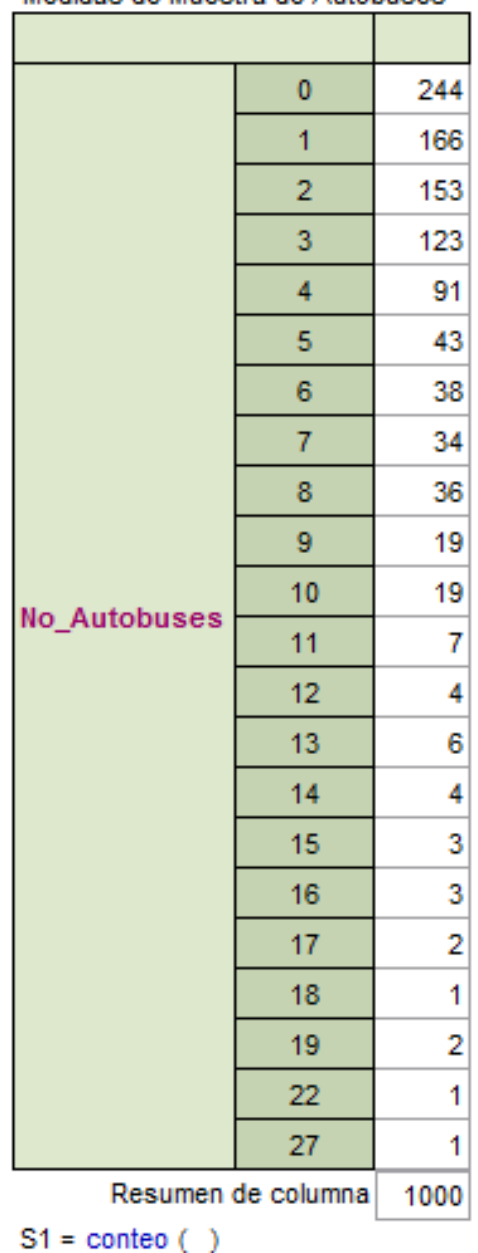


- ¿Cuántos autobuses crees que pueden llegar antes de que llegue el primero que te puede llevar a tu destino?

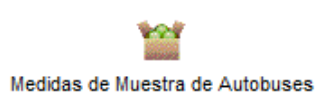

\begin{tabular}{|c|c|c|c|c|c|}
\hline \multicolumn{5}{|c|}{ Inspeccionar Medidas de Muestra de A... } & $\Sigma 3$ \\
\hline \multicolumn{2}{|c|}{ Casos Medidas } & Comentarios & Aparie... & Cat... & Rec... \\
\hline Medida & Valor & \multicolumn{4}{|c|}{ Fórmula } \\
\hline promedio & 3.026 & \multicolumn{4}{|c|}{ media (No_Autobuses) } \\
\hline
\end{tabular}

- ¿Cuál crees que será el máximo número de autobuses que llegarán antes de que llegue el autobús que te puede llevar a tu destino?

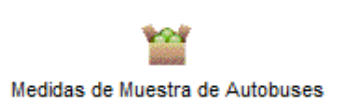

Inspeccionar Medidas de Muestra de A...ऍ
\begin{tabular}{|c|c|c|c|c|c}
\hline Casos & Medidas & Comentarios & Aparie... & Cat... & Rec. \\
\hline Medida & Valor & \multicolumn{2}{|c}{ Fórmula } \\
\hline máximo & 27 & máx (No_Autobuses) \\
\hline
\end{tabular}

- ¿Cuál es la probabilidad que entre los primeros cinco autobuses que lleguen se encuentre al menos uno que te lleve a tu destino?

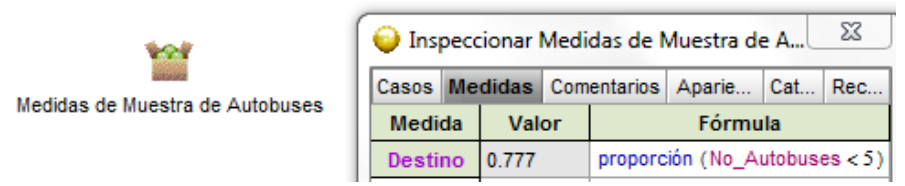

Solución teórica. Sea $X$ : el número de autobuses que llegan a la terminal antes del primero que te lleva a su destino. $X \sim G\left(p=\frac{3}{4}\right)$ donde su función de probabilidad está dada por $f_{X}(x)=(1-p)^{x} p$, con $x=0,1,2, \ldots$

$E(X)=\frac{p}{1-p}=\frac{\frac{3}{4}}{1-\frac{3}{4}}=3$, es decir que, en promedio, se espera que lleguen 3 buses antes de que llegue el primero que lo lleva a su destino.

Sea Y: el número de buses que lo llevan al destino de los cinco que llegan.

$Y \sim B\left(n=5, p=\frac{1}{4}\right)$ donde su función de probabilidad está dada por

$$
f_{Y}(y)=C(n, y) p^{y} q^{n-y}, \text { con } y=0,1,2, \ldots, 5
$$

$P(Y \geqslant 1)=\sum_{y=1}^{y=5} C(5, y)\left(\frac{1}{4}\right)^{y}\left(\frac{3}{4}\right)^{5-y}=\frac{781}{1024}=0.76270$ 


\subsubsection{Torres de electricidad}

Los pueblos A, B, C y D están enlazados por líneas de transmisión eléctrica entre A y B, A y C, B y C, y C y D. La planta generadora está en A (ver la figura). Durante una tormenta severa, la probabilidad que alguna línea en particular se caiga por defecto del clima es 0.2 y es independiente de cualquier línea. ¿Cuál es la probabilidad que el pueblo D tenga energía eléctrica después de la tormenta? ¿Qué es más probable que haya energía en el Pueblo D después de una tormenta severa o que no haya?

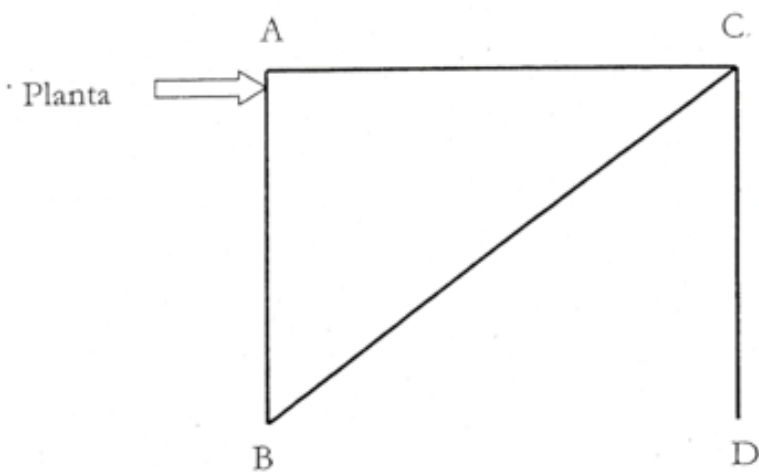

\subsubsection{Simulación en computadora}

1. Arrastre una colección y asígnele el nombre de Torres

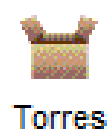

2. Arrastre la tabla correspondiente y escribe los siguientes atributos: LíneaAB, Línea_AC, LíneaBC, LíneaCD y PuebloD.

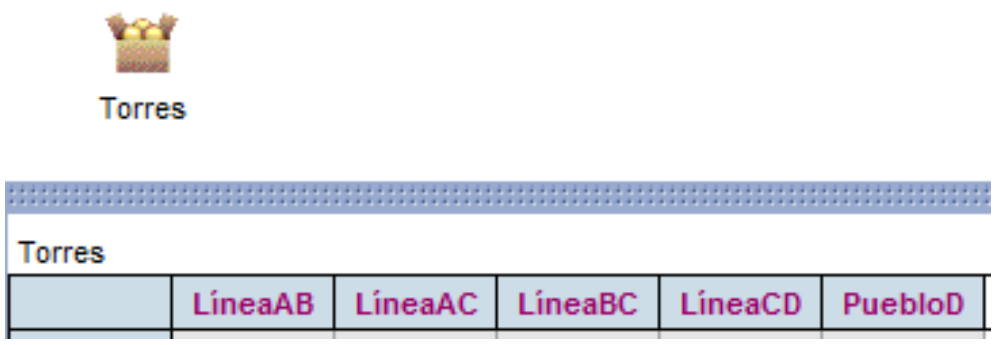

3. Asigne a cada Línea $(\mathrm{AB}, \mathrm{AC}, \mathrm{BC}$ y $\mathrm{CD})$ la fórmula: 


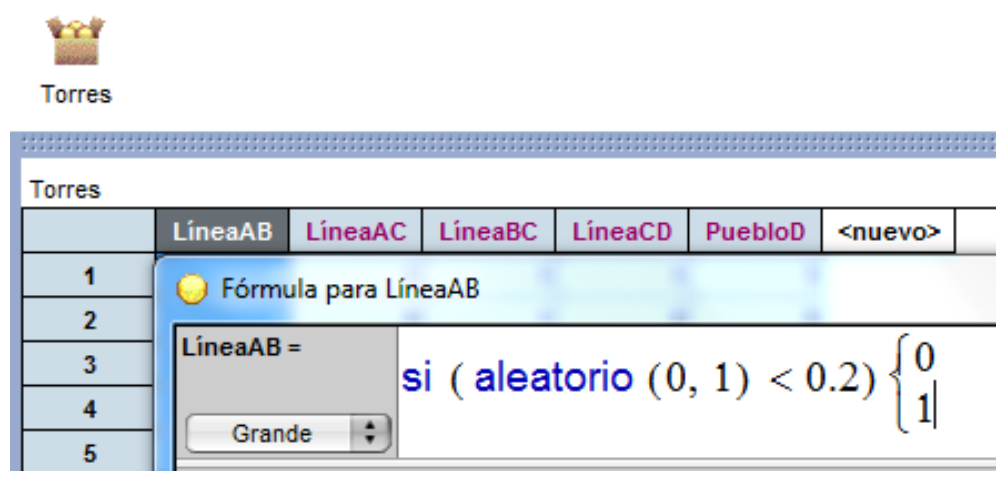

Donde 1 significa que la línea funciona perfectamente mientras que 0 significa que hay un defecto en la Línea.

Arrastre 1000 casos y analice algunos de los resultados.

4. En el pueblo D tendrá energía eléctrica si se obtiene un 1, en caso contrario un 0 de la siguiente forma:

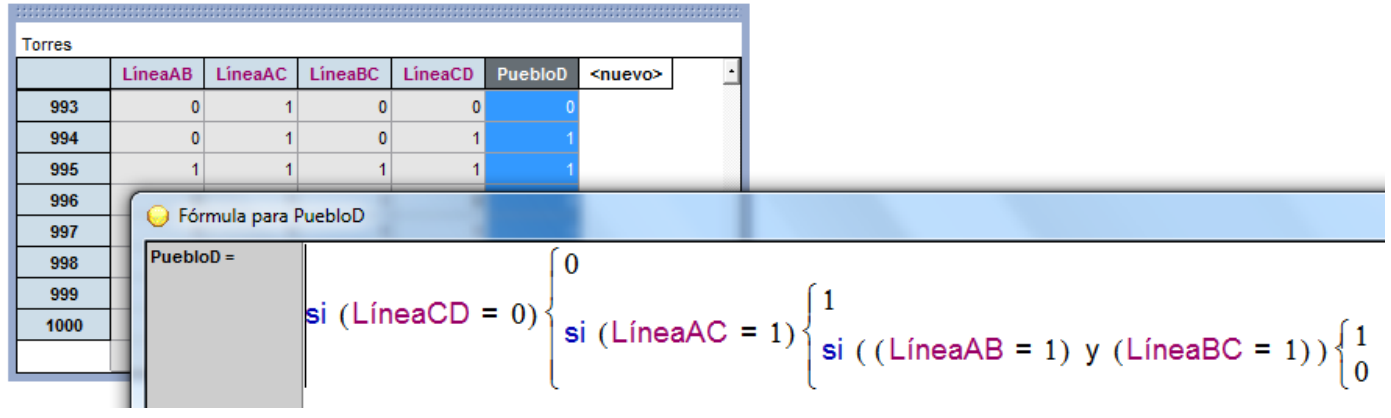

5. Construya una gráfica arrastrando el ícono de gráficas y colocando su eje horizontal el atributo PuebloD, a la vez que realizas el arrastre presiona la tecla shift.

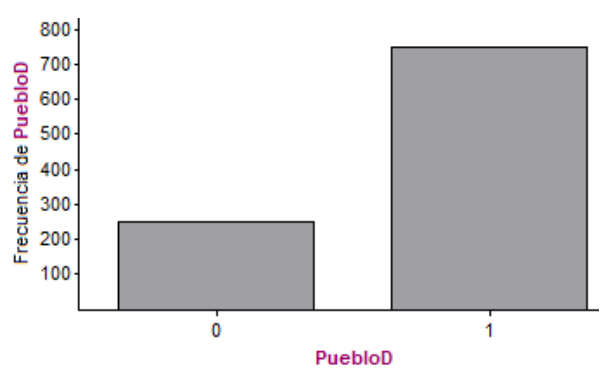

6. Arrastre el ícono de tabla resumen y coloque sobre esta el atributo PuebloD, manteniendo presionada la tecla shift durante el arrastre. 


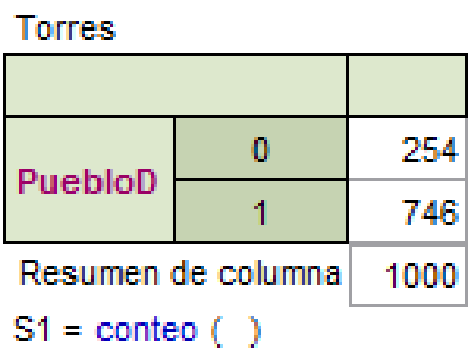

7. Observe la tabla anterior y contesta las preguntas que se plantearon al principio del problema. ¿Cuál es la probabilidad que el pueblo D tenga energía eléctrica después de una tormenta? ¿Qué es más probable que haya en el pueblo D después de una tormenta severa o que no haya?

Solución teórica. Sean $A_{1}$ : el evento de tener electricidad en las líneas $A C$ y $C D$

$A_{2}:$ el evento de tener electricidad en las líneas $A B, B C$ y $C D$

$D$ : el evento de tener electricidad en el pueblo $D$.

$P\left(A_{1}\right)=P(A C \cap C D)=\frac{4}{5} \cdot \frac{4}{5}=\frac{16}{25}$, por ser independientes.

$P\left(A_{2}\right)=P(A B \cap B C \cap C D)=\frac{4}{5} \cdot \frac{4}{5} \cdot \frac{4}{5}=\frac{64}{125}$, por ser independientes.

$P(D)=P\left(A_{1} \cup A_{2}\right)=P\left(A_{1}\right)+P\left(A_{2}\right)-P\left(A_{1} \cap A_{2}\right)$, por el principio de inclusión-exclusión

$=\left(\frac{4}{5}\right)^{2}+\left(\frac{4}{5}\right)^{3}-\left(\frac{4}{5}\right)^{4}=\frac{464}{625} \approx 0.7424$

O bien al calcular la probabilidad del complemento

$P(\neg D)=P[\neg C D \cup C D \cap(\neg A C \cap(\neg A B \cup \neg B C))]$

$=\frac{1}{5}+P[C D \cap((\neg A C \cap \neg A B) \cup(\neg A C \cap \neg B C))]$

$=\frac{1}{5}+P[(C D \cap \neg A C \cap \neg A B) \cup(C D \cap \neg A C \cap \neg B C)]$

$=\frac{1}{5}+\left[\frac{4}{5}\left(\frac{1}{5}\right)^{2}+\frac{4}{5}\left(\frac{1}{5}\right)^{2}-\frac{4}{5}\left(\frac{1}{5}\right)^{3}\right]$, por el principio de inclusión-exclusión

$=\frac{161}{625} \approx 0.2576$

Por lo que después de una tormenta severa es más probable que haya electricidad $(p=0.7424)$ a que no haya $(p=0.2576)$.

\subsubsection{Los signos del zodiaco}

Problema 1. Estimar la probabilidad de que en un grupo de 5 personas, al menos dos de ellas tengan el mismo signo del Zodiaco. (Hay 12 signos zodiacales y asumimos que cada signo es igualmente probable para cualquier persona). ¿Qué tal alta crees que es la probabilidad? 
Simulación en computadora.

1. De la barra de herramientas de Fathom arrastre una nueva colección y asígnale el nombre de Zodiaco.

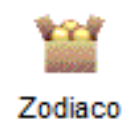

2. Arrastre la tabla correspondiente y defina el atributo Signos, entonces escriba los 12 signos zodiacales.

Zodiaco
Zodiaco
\begin{tabular}{|c|l|}
\hline & Signos \\
\hline 1 & Aries \\
\hline 2 & Cáncer \\
\hline 3 & Capricor... \\
\hline 4 & Leo \\
\hline 5 & Acuario \\
\hline 6 & Piscis \\
\hline 7 & Géminis \\
\hline 8 & Virgo \\
\hline 9 & Tauro \\
\hline 10 & Sagitario \\
\hline 11 & Libra \\
\hline 12 & Escorpión \\
\hline & \\
\hline
\end{tabular}

3. Seleccione la caja de Zodiaco haciendo clic sobre ella y del menú Colección seleccione Muestrear casos, lo cual significa que desea tomar una muestra de la población Zodiaco. Automáticamente aparece otra caja con la etiqueta Muestra de Zodiaco.

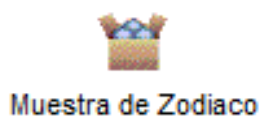

4. Active el inspector haciendo doble clic en la caja de Muestra de Zodiaco. Por omisión la muestra es de tamaño 10, cámbielo a 5 . Además, desactivar la animación para que sea más 
rápido el proceso de muestreo. En la pestaña Medidas defina la fórmula que le permitirá contar si entre las 5 personas hay al menos dos que tienen el mismo signo zodiacal, como se muestra a continuación:

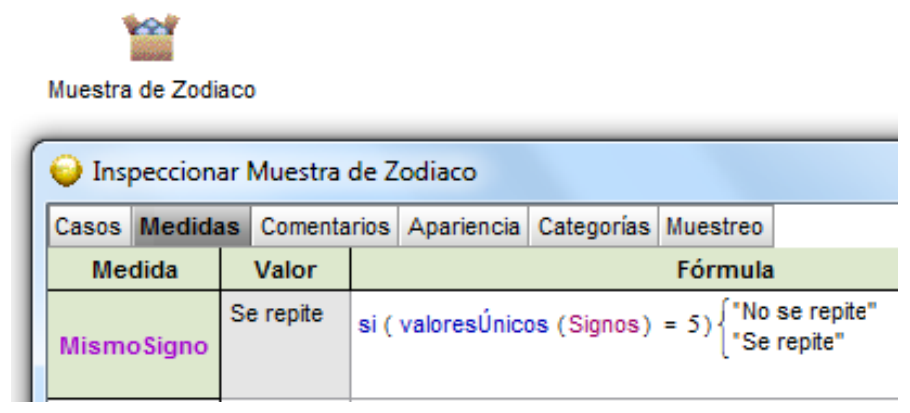

5. Active la caja Muestra de zodiaco y arrastra la tabla correspondiente, presione Muestrear más casos en el Inspector y aparecerá tablas similares a la siguiente:

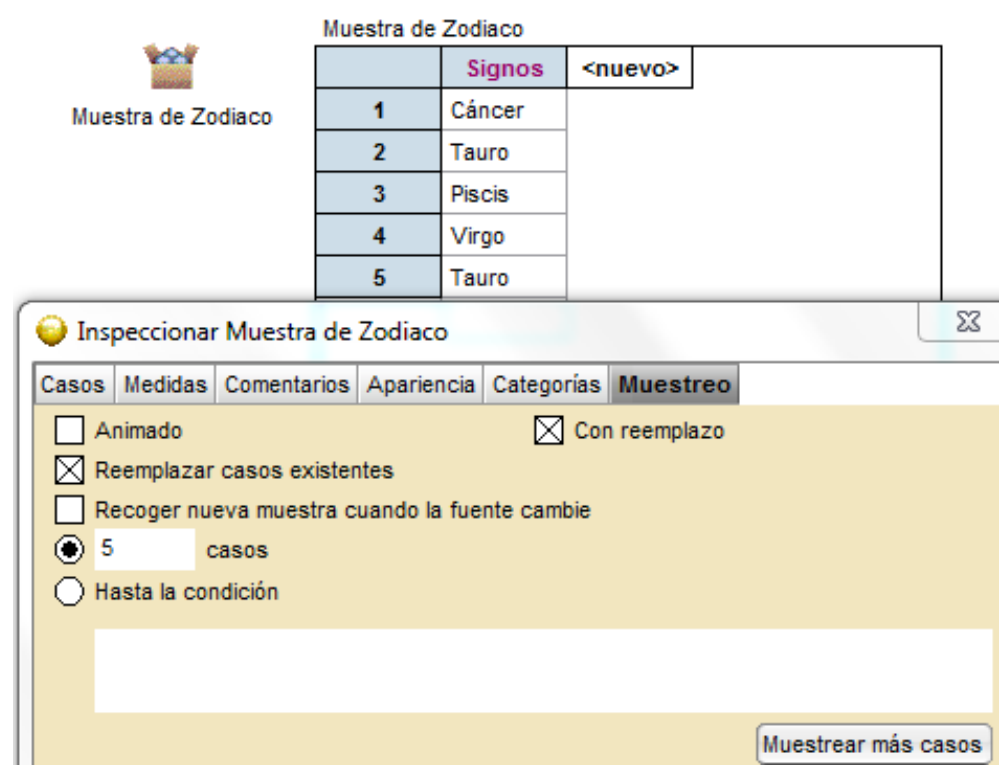

Se puede observar si existen o no al menos dos personas con el mismo signo zodiacal.

6. Activa la caja Muestra de Zodiaco y del menú Colección elige Recoger medidas, aparecerá entonces una nueva caja denominada Medidas de Muestra de casos.

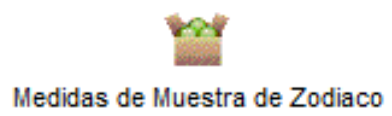

Modifique el inspector haciendo doble clic sobre la caja de Medidas de Muestra de Zodiaco y escriba 1000 medidas en lugar de 5, desactiva la animación, elimine los casos anteriores y presiona Recoger más medidas. Después active la caja de Medidas de Muestra de Zodiaco y arrastra la tabla correspondiente. 
7. Para graficar los datos anteriores, arrastra el ícono de gráfica y el atributo MismosSignos hacia el eje horizontal de la gráfica.

Medidas de Muestra de Zodiaco
\begin{tabular}{|c|l|l|}
\hline \multicolumn{3}{|c|}{ Medidas de Muestra de Zodiaco } \\
\hline 992 & Se repite & \\
\hline 993 & Se repite & \\
\hline 994 & Se repite & \\
\hline 995 & Se repite \\
\hline 996 & Se repite & \\
\hline 997 & No se re... \\
\hline 998 & No se re... \\
\hline 999 & No se re... \\
\hline 1000 & Se repite \\
\hline & \\
\hline
\end{tabular}

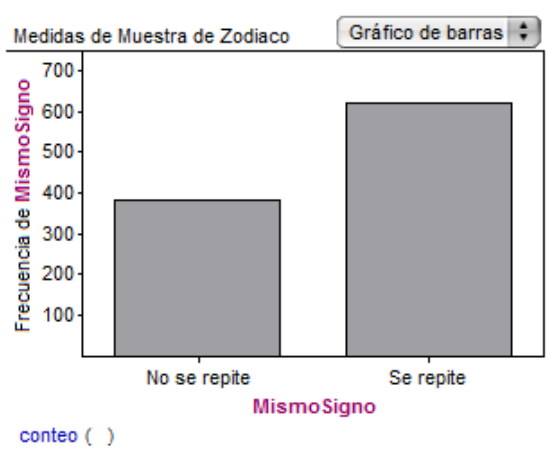

8. Para determinar la probabilidad empírica calcule en la pestaña de Medidas la proporción de signos que se repitieron de los 1000 experimentos. Así:

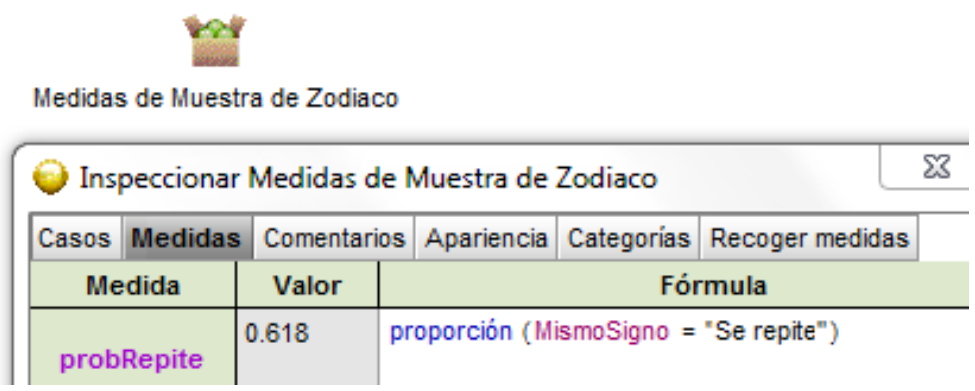

La probabilidad empírica de que en un grupo de 5 personas al menos dos tengan el mismo signo zodiacal es 0.618 en este ejemplo.

Solución teórica. Sea X: el evento de tener el mismo signo zodiacal al menos dos personas de las 5 personas seleccionadas.

$\bar{X}$ : el evento de que ninguna persona tenga el mismo signo zodiacal de las 5 personas seleccionadas.

$$
\begin{aligned}
& |U|=12^{5} \\
& |\bar{X}|=12 \cdot 11 \cdot 10 \cdot 9 \cdot 8 \\
& P(X)=1-P(\bar{X}) \\
& =1-\frac{12 \cdot 11 \cdot 10 \cdot 9 \cdot 8}{12^{5}}=\frac{89}{144} \approx 0.6181
\end{aligned}
$$


Problema 2. Ahora estamos interesados en estimar la probabilidad de que al menos una persona de un grupo de 5 personas, tenga el mismo signo zodiacal suyo.

\section{Simulación en computadora.}

El proceso va a cambiar del paso 4 en adelante.

1. Entre a la pestaña de Medidas y defina la fórmula que te permitirá contar si entre las 5 personas hay al menos una que tiene el mismo zodiacal que usted (supongamos que eres Cáncer).

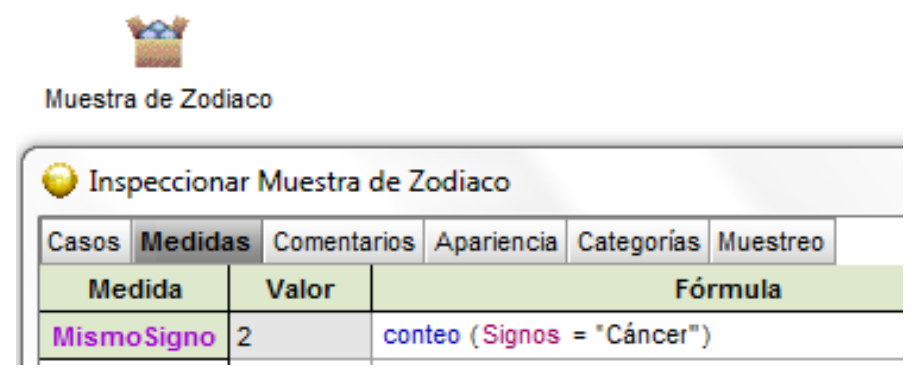

2. Modifique el inspector haciendo doble clic sobre la caja de Medidas de Muestra de Zodiaco y escriba 1000 medidas en lugar de 10, desactive la animación y presione Recoger más medidas. Después active la caja de Medidas de Muestra de Zodiaco y arrastra la tabla correspondiente.

3. Para graficar los datos anteriores, arrastra el ícono de gráfica y el atributo MismoSigno hacia el eje horizontal de la gráfica. También puedes agregar una tabla resumen con la proporción de personas que pueden tener el mismo signo que usted.
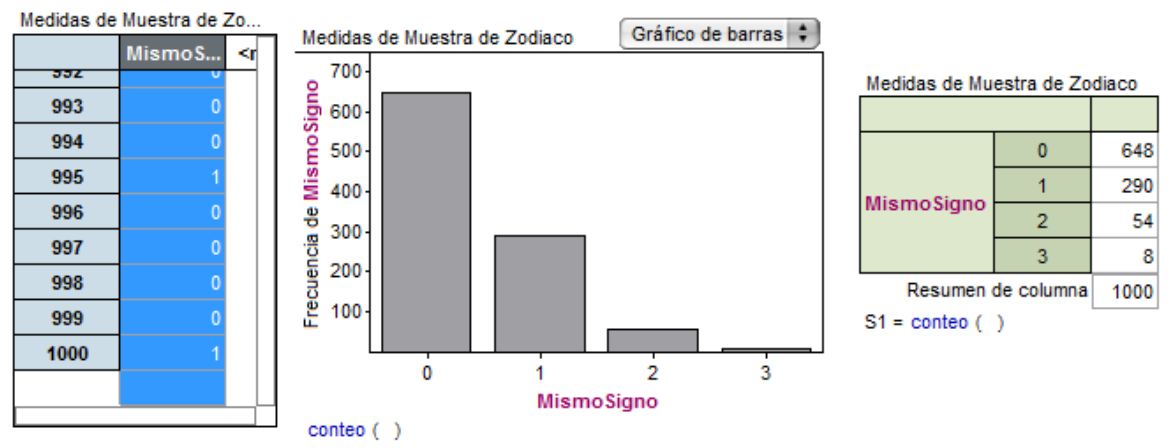

4. Para determinar la probabilidad empírica calcule en la pestaña de Medidas la proporción del Signo Cáncer que se repitió de los 1000 experimentos. Así:

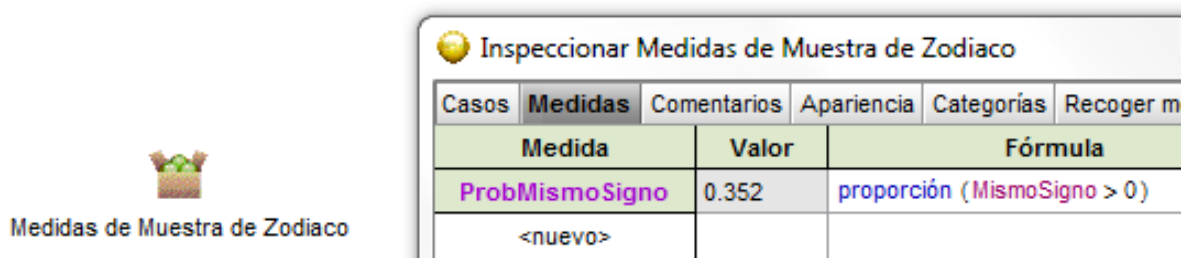


Solución teórica. Sea $X$ : el evento de tener el mismo signo zodiacal que uno de las 5 personas seleccionadas.

$\bar{X}$ : el evento de que ninguna persona tenga el mismo signo que uno de las 5 personas seleccionadas.

$$
\begin{aligned}
& |U|=12^{5} \\
& |\bar{X}|=11 \cdot 11 \cdot 11 \cdot 11 \cdot 11 \\
& P(X)=1-P(\bar{X}) \\
& =1-\frac{11^{5}}{12^{5}}=\frac{87781}{248832} \approx 0.3528
\end{aligned}
$$

\subsubsection{Familias chinas}

En un intento por reducir el crecimiento de la población, China ha instituido una política que permite a una familia tener descendencia sólo hasta cuando nazca la primera hija. Es decir, si el primer hijo es mujer ya no se les permite tener más hijos, sin embargo, si el primer hijo es hombre, se tiene la opción de tener más hijos si la familia así lo desea, hasta que nazca la primera hija. Supongamos que todas las familias chinas deciden tener hijos hasta que nazca la primer hija.

- ¿Cuántos hijos e hijas crees que en promedio vaya a tener cada familia china con la implementación de esta política?

- ¿Crees que con la implementación de esta política, en un tiempo razonable existiría la misma proporción de niños y niñas de China?, o ¿podría haber más de un sexo que de otro?

- ¿Qué crees que sea más probable? Que se tenga la primera hija en:

a) el primer nacimiento b) el segundo nacimiento c) el tercer nacimiento

\section{Simulación en computadora}

1. De la barra de herramientas de Fathom arrastra una caja colección y asígnele el nombre de Chinos

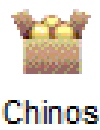

2. Arrastre la tabla correspondiente y al primer atributo asígnele el nombre de Hijos y escriba directamente $\mathrm{H}$ en el primer renglón y $\mathrm{M}$ en el segundo.

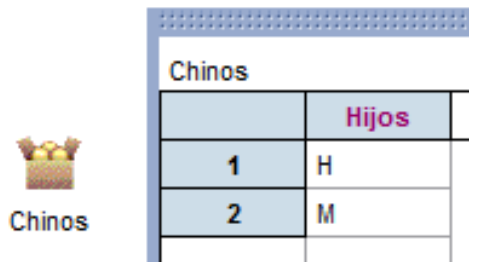


3. Seleccione la caja de Chinos haciendo clic sobre ella y del menú Colección seleccione Muestrear Casos, lo cual significa que desea tomar una muestra de la población Chinos. Automáticamente aparece otra caja con la etiqueta Muestra de Chinos.

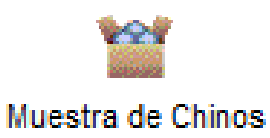

4. Active el inspector haciendo doble clic en la caja de Muestra de Chinos y modifíquelo como se muestra a continuación:

Por omisión el programa toma una muestra de tamaño 10, pero se puede cambiar, ya sea modificando el número de casos o seleccionado la condicional Hasta la condición, como la haremos en este caso tal como aparece en la figura.

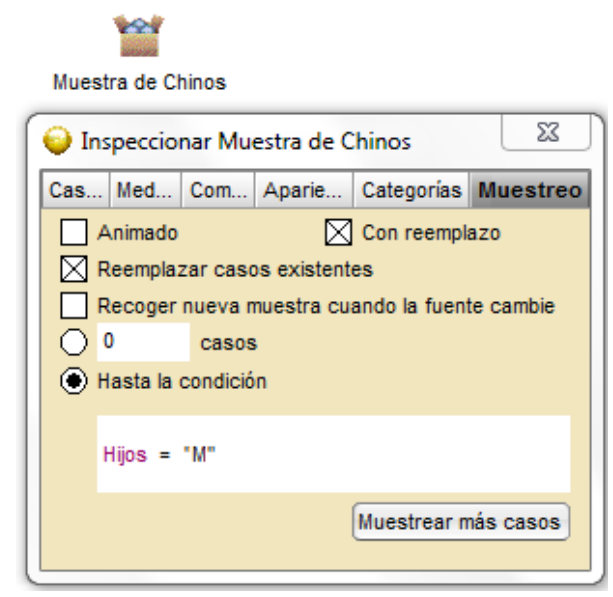

5. Arrastre la tabla correspondiente a la muestra tomada. Presione Ctrl Y para tomar muestras sucesivas.

6. Una vez que se han realizado muchas simulaciones nos interesa saber cómo se distribuye el número de hijos en cada familia (muestra). Active la colección Muestra de Chinos dando doble clic, de inmediato aparecerá el inspector, elige la pestaña Medidas y escribe NúmHijos que es la medida que queremos conocer. Haciendo doble clic en la casilla de Fórmula inmediatamente aparece el editor de fórmulas, escribe la fórmula conteo (Hijos) y presiona Aceptar, en este momento se genera el número de integrantes que tuvo una familia hasta la primera hija.

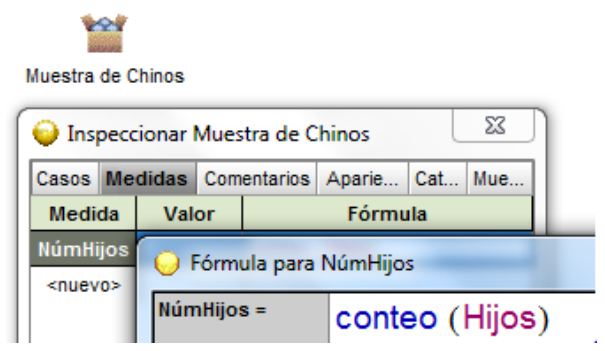


7. Active la colección Muestra de Chinos y del menú Colección elige Recoger medidas. Aparecerá entonces una nueva caja denominada Medidas de Muestra de Chinos

8. Arrastre y despliegue la tabla correspondiente. Por omisión se despliegan 5 medidas. Modifique el inspector haciendo doble clic sobre la caja de Medidas de Muestra de Chinos y escriba 1000 casos en lugar de 5, desactive la animación y presione Recoger más medidas.

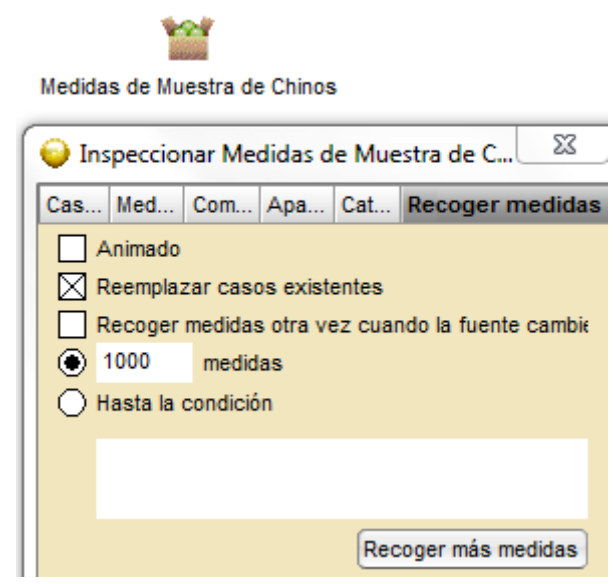

9. Active la caja Medidas de Muestra de Chinos y arrastre la tabla correspondiente. Para graficar los datos anteriores, coloque el ícono de gráfica en la hoja de trabajo y arrastre NúmHijos hacia el eje horizontal de la gráfica. Para obtener una gráfica de barras, al mismo tiempo que arrastre NúmHijos, oprima, sin soltar la tecla shift. Enseguida aparecerá una gráfica similar a la que se muestra enseguida.

\begin{tabular}{|c|c|c|}
\hline रणग & NúmHijos & <nuevo> \\
\hline 966 & 1 & \\
\hline 967 & 2 & \\
\hline 968 & 1 & \\
\hline 969 & 3 & \\
\hline 970 & 2 & \\
\hline 971 & 1 & \\
\hline 972 & 1 & \\
\hline 973 & 6 & \\
\hline 974 & 2 & \\
\hline
\end{tabular}

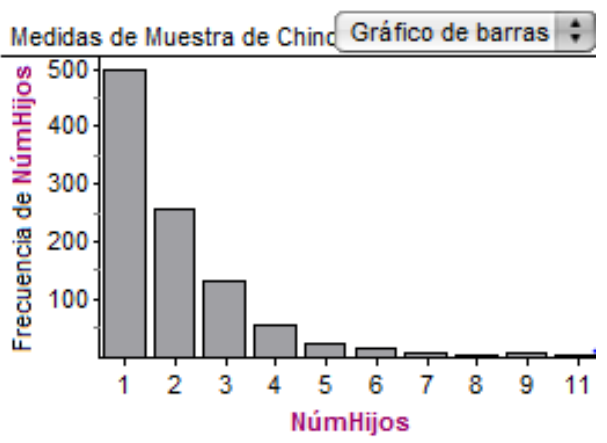

conteo ( )

10. Utilice el ícono de la tabla resumen y arrastre hacia ella el atributo NúmHijos. 


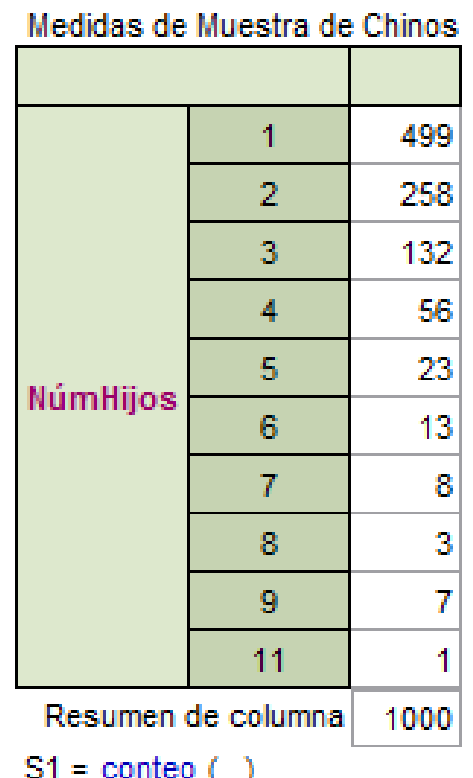

Observe la gráfica y la tabla para contestar las preguntas que se te plantearon al principio de la actividad.

- ¿Cuántos hijos e hijas crees que en promedio vaya a tener cada familia china con la implementación de esta política?

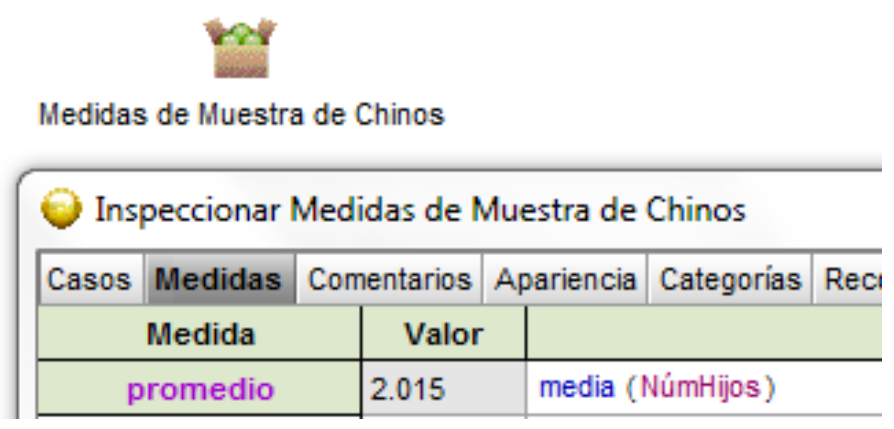

- ¿Crees que con la implementación de esta política, en un tiempo razonable existiría la misma proporción de niños y niñas de China?, o ¿podría haber más de un sexo que de otro?

\begin{tabular}{|c|c|c|c|c|c|}
\hline \multicolumn{6}{|c|}{ Medidas de Muestra de Chinos } \\
\hline \multicolumn{6}{|c|}{ Inspeccionar Medidas de Muestra de Chinos } \\
\hline \multirow[t]{2}{*}{ Casos } & Medidas & Comentarios & Apariencia & Categorias & Recoger $n$ \\
\hline & Medida & Valor & & & Fór \\
\hline \multicolumn{2}{|c|}{ promedio } & 2.015 & \multicolumn{3}{|c|}{ media (NúmHijos) } \\
\hline \multicolumn{2}{|c|}{ CuentaHombres } & 1015 & \multicolumn{3}{|c|}{ suma (NúmHijos) -1000} \\
\hline
\end{tabular}


- ¿Qué crees que sea más probable? Que se tenga la primera hija en:

a) el primer nacimiento b) el segundo nacimiento c) el tercer nacimiento

Solución teórica. Sea X: el número de hijos que tiene cada familia en China (núm de hijos hasta antes de tener la primer hija)

$X \sim G\left(p=\frac{1}{2}\right)$ donde su función de probabilidad está dada por

$$
f_{X}(x)=(1-p)^{x} p, \operatorname{con} x=0,1,2, \ldots
$$

$E(X)=\frac{p}{1-p}=\frac{\frac{1}{2}}{1-\frac{1}{2}}=1$, es decir que, en promedio, cada familia va tener a dos hijos, pues en promedio debe tener un hijo antes de tener su primer hija.

$$
\begin{gathered}
P(X=0)=\frac{1}{2} \\
P(X=1)=\left(\frac{1}{2}\right)^{1} \frac{1}{2}=\frac{1}{4} \\
P(X=2)=\left(\frac{1}{2}\right)^{2} \frac{1}{2}=\frac{1}{8}
\end{gathered}
$$

Es más probable obtener la primer hija en el primer nacimiento.

\subsubsection{Encuentro entre amigos}

Dos amigos han decidido encontrarse en un restaurante entre 8 y 9 de la noche. Cada uno de ellos entra al restaurante en forma aleatoria en cualquier minuto de ese periodo y esperan 16 minutos máximo y luego se retiran. ¿Cuál será la probabilidad de que se encuentren?

\section{Simulación en computadora}

1. Construya una colección llamada Amigos y genera la tabla con las siguientes columnas:

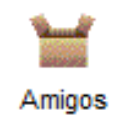

Amigos
\begin{tabular}{|l|l|l|l|l|}
\hline & LlegadaAmigo1 & LLegadaAmigo 2 & Diferencia & EncuentrooNO \\
\hline & & & & \\
\hline
\end{tabular}

2. Asigne un tiempo aleatorio de llegada del primer amigo y el segundo amigo entre las 8 y las 9 .

3. Obtenga la diferencia entre los tiempos de llegada de los dos amigos. 
4. Defina si los amigos se encuentran o no según la diferencia de los tiempos de llegada.

5. Aumenta a 1000 el número de llegadas de los amigos y calcula la probabilidad empírica de que los dos amigos se encuentren.

6. ¿Cuál será la probabilidad de que no se encuentren?

7. ¿Cuál será la probabilidad de que se encuentren a la entrada del restaurante?

8. ¿Cómo podrías calcular la probabilidad teórica de que los amigos se encuentren?

Solución teórica. Se cumple que el espacio muestra es $\Omega=\left\{w=\left(w_{1}, w_{2}\right): 0 \leq w_{1} \leq 60 \wedge 0 \leq w_{2} \leq 60\right\}$

Sea $B$ : el evento de que los amigos se encuentran.

Así que $B=\left\{x \in \Omega: w_{1}-w_{2} \leq 16 \vee w_{2}-w_{1} \leq 16\right\}$

$B=\left\{x \in \Omega: w_{1}-16 \leq w_{2} \vee w_{2} \leq 16+w_{1}\right\}$

El área del espacio muestral es $A_{\Omega}=60^{2}=3600$

El área del complemento de $B$ es $A_{\bar{B}}=44^{2}=1936$

Gráficamente

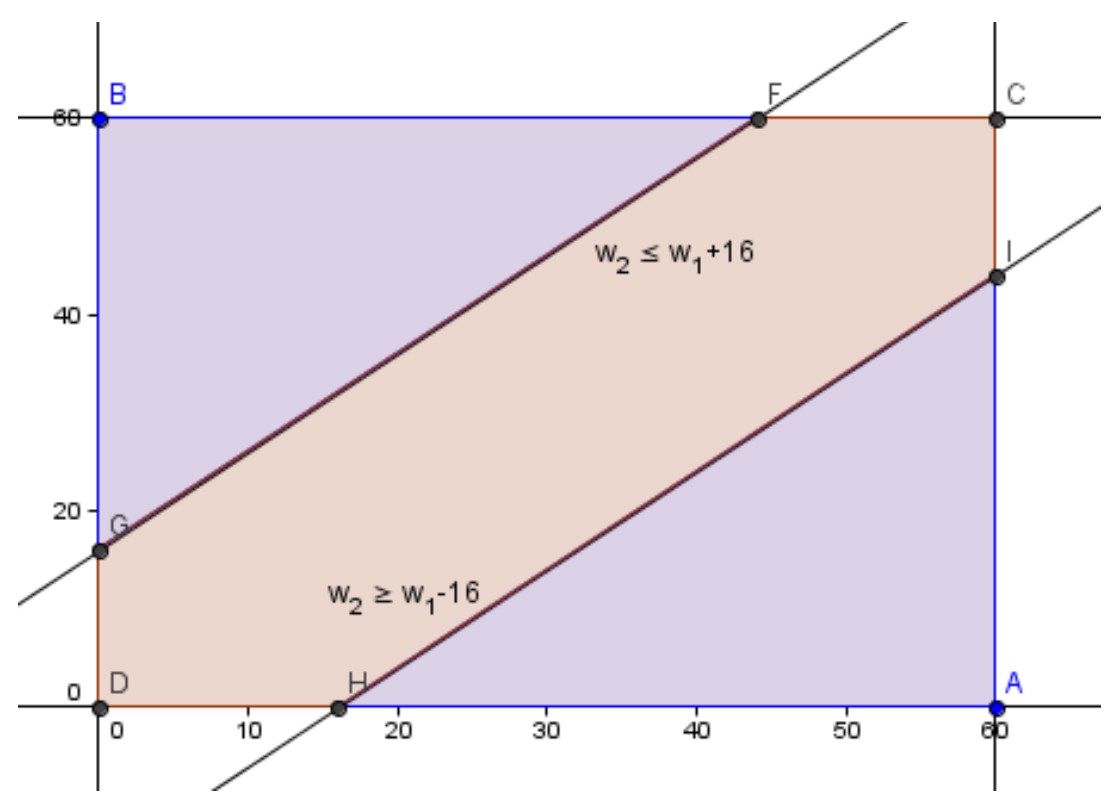

$P(B)=1-P(\bar{B})$

$P(B)=1-\frac{1936}{3600}=\frac{104}{225} \approx 0.4622$ 


\subsubsection{El banco}

Un banco de la ciudad sólo abre dos ventanillas para atender a sus clientes. El número de clientes que llega al banco varía entre 0 y 5 por minuto. Los clientes forman una línea y la persona de adelante pasa a la primera ventanilla disponible. En las ventanillas se atiende una persona por minuto. Diseñe una simulación y registre el número de personas en la línea de espera al final de cada minuto. Use una tabla como la siguiente:

\begin{tabular}{|c||c|c||c|}
\hline Minuto & $\begin{array}{c}\text { Núm de clientes que } \\
\text { llegan }\end{array}$ & $\begin{array}{c}\text { Núm de clientes } \\
\text { esperando en línea }\end{array}$ & $\begin{array}{c}\text { Tiempo de espera última } \\
\text { persona (min) }\end{array}$ \\
\hline 1 & 3 & 1 & 1 \\
\hline 2 & 4 & 3 & 2 \\
\hline
\end{tabular}

- ¿Cuál es la longitud de la fila después de cinco minutos?

- ¿Cuál es el tiempo que una persona tiene que esperar si llegó en el minuto 10?

- ¿Cuántas veces el tiempo de espera se redujo a cero?

- ¿Cuál es el promedio de personas esperando sobre el período de 20 minutos?

- Si tú fueras gerente de un banco ¿incrementarías o disminuirías el número de ventanillas?

\section{Simulación en computadora}

1. Construya en Excel una tabla que contenga las siguientes columnas:

\begin{tabular}{|c|c|c|c|c|}
\hline$\Delta$ & A & B & C & D \\
\hline 1 & Minuto & NúmClientesArriban & NúmClientesenLínea & TiempoEsperaúltimaPersona \\
\hline 2 & 0 & 0 & 0 & 0 \\
\hline
\end{tabular}

En el minuto cero aún no han llegado clientes al banco.

2. Asignar un número aleatorio entre cero y cinco a la columna NúmClientesArriban en cada minuto que transcurre. Este número será la cantidad de personas que arriban al banco por minuto. Así:

\begin{tabular}{|c|c|c|c|c|}
\hline \multicolumn{2}{|c|}{ COVARIA... } & $: \times \vee f_{x}$ & \multicolumn{2}{|c|}{$=$ ALEATORIO.ENTRE $(0,5)$} \\
\hline 4 & A & B & C & $\mathrm{D}$ \\
\hline 1 & Minuto & NúmClientesArriban & NúmClientesenLínea & TiempoEsperaúltimaPersona \\
\hline 2 & 0 & 0 & 0 & 0 \\
\hline 3 & 1 & $=$ ALEATORIO.ENTRE $(0$, & & \\
\hline
\end{tabular}

3. Se contabilizan las personas que permanecen haciendo fila con la siguiente fórmula asignada a NúmClientesenLínea: 


\begin{tabular}{|c|c|c|c|c|}
\hline \multicolumn{2}{|c|}{ COVARIA... $\quad$} & $\vdots \times \vee f_{x}$ & \multicolumn{2}{|c|}{$=\mathrm{SI}((\mathrm{B} 3+\mathrm{C} 2)<=2,0,(\mathrm{~B} 3-2)+\mathrm{C} 2)$} \\
\hline 2 & A & B & C & D \\
\hline 1 & Minuto & NúmClientesArriban & NúmClientesenLínea & TiempoEsperaÚltimaPersona \\
\hline 2 & 0 & $\underline{0}$ & 이 & 0 \\
\hline 3 & 1 & 5 & $=\mathrm{SI}((\mathrm{B} 3+\mathrm{C} 2)<=2,0,(\mathrm{~B} 3-2$ & $2)+(2)$ \\
\hline
\end{tabular}

4. Se calcula el tiempo de espera de la última persona que está haciendo fila asignando la fórmula:

\begin{tabular}{|c|c|c|c|c|}
\hline \multicolumn{2}{|c|}{ COVARIA... $\quad$} & $: \times \vee f_{x}$ & \multicolumn{2}{|c|}{$=\mathrm{REDONDEAR}(\mathrm{C} 3 / 2,0)$} \\
\hline$\Delta$ & A & B & $\mathrm{C}$ & D \\
\hline 1 & Minuto & NúmClientesArriban & NúmClientesenLínea & TiempoEsperaÚltimaPersona \\
\hline 2 & 0 & 0 & 0 & 0 \\
\hline 3 & 1 & 5 & & $=$ REDONDEAR $(\mathrm{C} 3 / 2,0)$ \\
\hline
\end{tabular}

5. Arrastre estas fórmulas hasta el minuto 500 para determinar lo que pasa después de ocho horas. Calcule el promedio de clientes que arriban en los 500 minutos.

\begin{tabular}{|c|c|c|c|c|}
\hline \multicolumn{2}{|c|}{ COVARIA... } & \multicolumn{3}{|c|}{ =PROMEDIO(B2:B502) } \\
\hline 4 & A & B & C & $\mathrm{D}$ \\
\hline 1 & Minuto & NúmClientesArriban & NúmClientesenLínea & TiempoEsperaúltimaPersona \\
\hline 2 & 0 & 0 & 0 & 0 \\
\hline 3 & 1 & 2 & 0 & 0 \\
\hline 4 & 2 & 5 & 3 & 2 \\
\hline 500 & 498 & 4 & 260 & 130 \\
\hline 501 & 499 & 2 & 260 & 130 \\
\hline 502 & 500 & 0 & 258 & 129 \\
\hline 503 & & & & \\
\hline 504 & & & Promedio arriban & =PROMEDIO(B2:B502) \\
\hline
\end{tabular}

Observe el TiempoEsperaÚltimaPersona a los 500 minutos (8 horas y 20 minutos). ¿Si tú fueras gerente de un banco, incrementarías o disminuirías el número de cajeros en las ventanillas?

6. Obtenga el número de clientes que permanecerían en la línea después de los 500 minutos.

\begin{tabular}{|r|r|r|r|r|}
\hline 500 & 498 & 4 & 260 & 130 \\
\hline 501 & 499 & 2 & 260 & 130 \\
\hline 502 & 500 & $0 \mid$ & 258 & 129 \\
\hline 503 & & & & \\
\hline 504 & & Promedio arriban & & 2.510978044 \\
\hline 505 & & Total de los que esperan & =C502 & \\
\hline
\end{tabular}

7. Halle el número esperado de personas en línea después de esos 500 minutos. 


\begin{tabular}{|c|c|c|c|c|}
\hline \multicolumn{2}{|c|}{ COVARIA... - } & \multicolumn{3}{|c|}{$=($ D504-2)*A502 } \\
\hline$\Delta$ & A & B & $\mathrm{C}$ & D \\
\hline 1 & Minuto & NúmClientesArriban & NúmClientesenLínea & TiempoEsperaÚltimaPersona \\
\hline 2 & c & 0 & 0 & 0 \\
\hline 3 & 1 & 2 & 0 & 0 \\
\hline 4 & 2 & 5 & 3 & 2 \\
\hline 500 & 498 & 4 & 260 & 130 \\
\hline 501 & 499 & 2 & 260 & 130 \\
\hline 502 & 500 & 0 & 258 & 129 \\
\hline 503 & & & & \\
\hline 504 & & & Promedio arriban & 2.510978044 \\
\hline 505 & & & Total de los que esperan & 258 \\
\hline 506 & & & Núm esperado de personas en línea & $=(\mathrm{D} 504-2) * \mathrm{~A} 502$ \\
\hline
\end{tabular}

8. Se puede observar que después de un largo tiempo, el total de personas que deben esperar en línea corresponde aproximadamente a la mitad del tiempo que ha transcurrido desde que abrió las puertas del banco

\begin{tabular}{|l|r|}
\hline Promedio arriban & 2.510978044 \\
\hline Total de los que esperan & 258 \\
\hline Núm esperado de personas en línea & 255.489022 \\
\cline { 2 - 2 }
\end{tabular}

Solución teórica. Sea X: el número de personas que llegan por minuto.

$X \sim U[0,5]$ donde su esperanza está dada por

$$
E(X)=\frac{a+b}{2}=\frac{0+5}{2}=\frac{5}{2}=2.5
$$

Es decir que, en promedio, deben llegar 2.5 clientes por minuto al banco.

Como cada ventanilla atiende a una persona por minuto, entonces se reduce en dos el número de personas que por minuto esperan en la fila. Así que el número esperado de personas en la fila después de un tiempo $t$ está dado por

$$
t \cdot(E(X)-2)
$$

Al cabo de $t=500$ minutos, que corresponde a 8 horas con 20 minutos, el número de clientes que deben esperar en la fila a de ser:

$t \cdot(E(X)-2)=500 \cdot \frac{1}{2}=250$.

\subsection{Conclusión}

Se concuerda con el Ministerio de Educacion Pública [4] cuando se refiere a que la hoja de cálculo es una poderosa herramienta que permite al individuo utilizarla como instrumento de análisis en el proceso repetitivo de experimentos, con el fin de llegar a la solución empírica de problemas probabilísticos.

Simulación de problemas probabilísticos . Greivin Ramírez

Derechos Reservados (C) 2015 Revista digital Matemática, Educación e Internet (http://tecdigital.tec.ac.cr/revistamatematica/) 
El proceso previo de creación de la simulación es fundamental en el desarrollo de pensamiento del individuo, debido a que debe plantearse un esquema lógico de diseño que lo lleve a modelar la situación planteada. Además, las estructuras básicas de programación empleadas permitirán que cada persona ponga en evidencia razonamientos cognitivos de nivel superior al tratar de dar solución a problemas de esta índole.

Por último, se sugiere, que en cualquier nivel educativo, los estudiantes utilicen este tipo de estrategias de simulación mediante paquetes computaciones con el fin de brindar solución a problemas sin tener, necesariamente que acudir, a las pruebas o contenidos matemáticos formales que respalden su solución, pues se sabe que muchas veces los estudiantes no le encuentran sentido o les abruma su ideología.

Este trabajo fue el resultado de una propuesta presentada en el seminario "Tópicos de matemática aplicada" desarrollado por la Escuela de Matematica del Instituto Tecnológico de Costa Rica. Este seminario tenía por objetivos: estudiar algunas de las técnicas más comúnmente usadas en el análisis y/o solución de problemas aplicados, promover el uso de la matemática aplicada y fomentar el trabajo interdisciplinario.

\section{Bibliografía}

[1] Ben Z-vi, D. y Garfield, J. (2004). Research on reasoning about variability: a forward. Statistic Education Research Journal, 3 (2), 4-6.

[2] Burrill, G. (2002). Simulation as a tool to develop statistical understanding. En B. Phillips (Ed). Proceedings of the Sixth International Conference on Teaching Statistics.Cape Town South Africa.

[3] Inzunsa, S. (2006). Significados que estudiantes universitarios atribuyen a las distribuciones muestrales en un ambiente de simulación computacional y estadística dinámica. Tesis doctoral no publicada. CINVESTAV-IPN. México.

[4] Ministerio de Educación Pública (MEP). (2012). Reforma Curricular en Ética, Estética y Ciudadanía. Programas de Estudio de Matemática. I y II Ciclo de Educación Primaria, III Ciclo de Educación General Básica y Diversificada. Informe Técnico. Ministerio de Educación Pública, San José, Costa Rica. Extraído el 01 de Agosto, 2014 en: http: //www. mep.go .cr/downloads/ RecursosTecnologicos/Programasmatematica.pdf

[5] Sanabria, G. (2013). Simulación en Excel de variables aleatorias discretas, Memorias del VII Congreso Iberoamericano de Educación Matemática (VII CIBEM). Montevideo, Uruguay.

[6] Shaughnessy, M. (1992). Research in Probability and Statistics: Reflections and Directions. En Grouws, D. A.(Ed.). Handbook of Research on Mathematics Teaching and Learning. New York. Macmillan Publishing Company, pp. 465-494.

Simulación de problemas probabilísticos. Greivin Ramírez

Derechos Reservados @ 2015 Revista digital Matemática, Educación e Internet (http://tecdigital.tec.ac.cr/revistamatematica/) 